\title{
SÍNTESE DE COPOLÍMEROS EM BLOCO COM PROPRIEDADES LÍQUIDO CRISTALINAS
}

\author{
Maria Rita Guinancio Coelho e Ailton de Souza Gomes* \\ Instituto de Macromoléculas Prof ${ }^{\mathrm{a}}$. Eloisa Mano, Universidade Federal do Rio de Janeiro, CP 68525, 21941-972 Rio de Janeiro \\ - RJ, Brasil
}

Recebido em 17/2/06; aceito em 31/5/06; publicado na web em 22/2/07

\begin{abstract}
SYNTHESIS OF LIQUID CRYSTALLINE BLOCK COPOLYMERS. The principal techniques for the synthesis of liquid crystalline block copolymers are reviewed. The syntheses are done by living/controlled free radical chain polymerization. The copolymers display an amorphous continuous phase and a discontinuous liquid crystalline phase (LC). The presence of oxypropylenic segments disturbs the range of mesophase transitions at lower temperatures. This behavior is not observed when styrenic segments are employed and suggests that the liquid crystalline behavior can be modified in block copolymers to show mesophases at higher and lower temperatures according to the flexibility of the chain segment that is present.
\end{abstract}

Keywords: block copolymer; liquid crystals; polymerization.

\section{INTRODUÇÃO}

A síntese de copolímeros em bloco líquido cristalinos foi primeiramente relatada ao final da década de 80 .

Ao que parece, apesar da literatura ${ }^{1,2}$ citar o trabalho de Adams e Gronski ${ }^{3}$, em 1989, como o pioneiro na síntese de copolímeros em bloco líquido cristalinos, Auman e Percec ${ }^{4}$, em 1988, foram os primeiros pesquisadores a sintetizar este tipo de substância. Desde então, uma série de trabalhos que envolvem rotas para produção dos mesmos foram sendo apresentados ${ }^{5-12}$. Pesquisadores italianos da Università di Piza e da Università di Bologna foram os que mais contribuíram para o desenvolvimento dessa área de pesquisa.

Os copolímeros são macromoléculas compostas por duas ou mais unidades estruturais diferentes, que podem se distribuir estatisticamente ente si ou de forma a gerar segmentos. Os copolímeros em bloco pertencem a esta última classe e seus segmentos são formados por uma sequiência linear de um tipo de unidade estrutural (mero) do tipo A, quimicamente ligada a uma outra seqüência linear de um mero do tipo B. Essas seqüências lineares são chamadas de blocos.

De acordo com a organização dos blocos na cadeia, os copolímeros em bloco são classificados como sendo do tipo radial (estrelado) ou do tipo seqüencial (linear). Esse último tipo, por sua vez, pode ser constituído por diblocos, triblocos ou ainda, por multiblocos.

Os copolímeros em bloco líquido cristalinos são copolímeros onde um dos segmentos é, necessariamente, formado por meros com características mesogênicas, isto é, contendo um agrupamento de átomos que apresentam uma geometria tal que resulte na polarizabilidade e anisotropia dielétrica típicas neste agrupamento de átomos que, por sua vez, confere a propriedade líquido cristalina (LC) ao copolímero².

Como as definições de propriedade LC, estado LC, unidades mesogênicas, mesofase, entre outros relacionados a polímeros LC encontram-se detalhadamente descritas em trabalhos anteriores ${ }^{13,14}$, não serão abordadas nesta revisão mas, é preciso destacar, que as unidades mesogênicas que compõem o segmento com características LC podem ordenar-se tanto de maneira seqüencial no segmento polimérico, gerando os chamados copolímeros em bloco LC de

*e-mail: asgomes@ima.ufrj.br cadeia principal ou podem estar lateralmente ligadas nos meros que compõem o bloco, formando os copolímeros em bloco LC de cadeia lateral.

A literatura aponta trabalhos de revisão que discutem as propriedades ${ }^{15-17}$ de copolímeros em bloco LC, assim como as metodologias empregadas para síntese dos mesmos ${ }^{2,18,19}$.

Na preparação desse tipo de substância faz-se necessária primeiramente a síntese dos núcleos mesogênicos que formarão o segmento responsável pelas propriedades LC, pois, via de regra, não se encontram comercialmente disponíveis e sua produção às vezes exige rotas com um número significativo de etapas, que pode chegar até a comprometer tanto o rendimento quanto a pureza do produto final. Todavia, esta parte será discutida oportunamente.

A principal dificuldade a ser contornada nas sínteses de copolímeros em bloco LC recai no fato de que, para os mesógenos apresentarem a requerida anisotropia dielétrica e polarizabilidade, é preciso a presença de grupos funcionais específicos que, por sua vez, podem ser bastante reativos e por isso vir a reagir com o grupo terminal da cadeia propagante durante a reação de polimerização. Este comportamento afeta a etapa de crescimento da cadeia, podendo resultar, muitas vezes, na produção de um material com baixo valor de massa molar assim como a sua larga distribuição, afetando o comportamento físico-mecânico do polímero final.

É sabido que os copolímeros em bloco não-LC, quando formados por segmentos quimicamente incompatíveis podem apresentar, no estados sólido, separação de fases perceptível somente em escala molecular ${ }^{2}$. Nos copolímeros em bloco LC especificamente, essa separação de fases ocorre devido à incompatiblidade entre o bloco LC e o bloco não-LC quando na mesofase.

A ligação do segmento LC com um segmento não-LC (amorfo), na forma de blocos, pode originar uma vasta faixa de combinações de propriedades e de estruturas possíveis, tornando essa classe de material interessante devido ao aparecimento simultâneo de dois tipos de ordenamento na mesofase. Um deles é oriundo da incompatibilidade que, quase sempre, ocorre entre blocos amorfos não compatíveis forçando a separação de fases, como no caso geral de copolímeros em bloco ordinários. O outro ordenamento, resultante da auto-orientação dos mesógenos, leva à formação de uma estrutura organizada no bloco LC, assim como ocorre no caso de homopolímeros LC análogos ${ }^{2,20}$. 
Sendo assim, os copolímeros em bloco LC são capazes de se organizar em microfases no estado sólido, isto é, numa estrutura com fases separadas em escala microscópica ${ }^{3}$. Tal característica permite que estes materiais já sejam empregados como indutores de reforço de polímeros amorfos tradicionais, assim como agentes compatibilizantes de misturas de polímeros LC com polímeros convencionais, entre outras. Ainda são capazes de permitir o desenvolvimento de novos materiais com propriedades previamente planejadas e uma variedade de aplicações, em particular para aplicações eletro-ópticas ${ }^{21-23}$.

Na síntese de copolímeros LC é importante que a reação ocorra de maneira controlada, já que o valor da massa molar assim como sua distribuição são responsáveis pelo tipo e tamanho das microfases formadas que, por sua vez, afetam de forma significativa as transições LC-isotrópicas².

A literatura cita alguns mecanismos e estratégias para síntese de copolímeros em bloco com propriedades LC $^{3,13,24-33}$. Dentre elas podem ser destacadas a reação de modificação química de copolímeros em bloco não-LC (matrizes copoliméricas), na qual um dos blocos contém grupamentos funcionais capazes de reagir com um grupo reativo ligado a uma unidade mesogênica ${ }^{3,13,24}$ a fotopolimerização de ésteres mesogênicos metacrílicos com catalisadores à base de porfirina de alumínio ${ }^{25}$; a copolimerização catiônica-radical de monômeros nucleofílicos e vinílicos em dois estágios ${ }^{22} \mathrm{e}$ as polimerizações vivas ${ }^{26-33}$.

Este último tipo de polimerização pode ser ainda subdividido nas seguintes polimerizações: por transferência de grupo (GTP) de monômeros $\alpha, \beta$-insaturados, usando acetais de silil-cetona como iniciador ${ }^{26}$; catiônica de éteres vinílicos mesogênicos ${ }^{27}$; aniônica entre o monômero de estireno, ou de metacrilato de metila ou de outros e um (met)acrilato líquido cristalino ${ }^{28,29} \mathrm{e}$, nas vias radicais livres "vivas", onde o que ocorre na realidade é um grande controle sobre a distribuição de massa molar, durante a reação de síntese.

Essas reações ou são iniciadas por um macroagente de transferência ${ }^{30}$ ou mediadas tanto por organodissulfetos (INIFERTER) $)^{31}$ quanto por nitróxidos estáveis (TEMPO) ${ }^{32}$ ou ainda, podem ocorrer por transferência atômica por meio de um metal de transição $(\mathrm{ATRP})^{33}$.

O objetivo do presente trabalho é destacar os resultados do empenho de pesquisadores, desde 1993, no Instituto de Macromoléculas Profa. Eloisa Mano, na síntese de copolímeros em bloco com propriedades LC. A produção dos copolímeros foi feita tanto pela introdução de segmentos orgânicos, que apresentam comportamento líquido cristalino em uma cadeia polimérica do tipo amorfa $^{13}$, quanto por policondensação de monômeros tipicamente líquido cristalinos ${ }^{34,35} \mathrm{e}$, ainda, por poliadições "vivas" via radicais livres de monômeros LC com monômeros ou macromonômeros amorfos $^{1,21,36}$. Entretanto, nesta revisão serão abordadas somente as metodologias centradas em poliadições "vivas".

\section{SÍNTESES DE COPOLÍMEROS EM BLOCO COM PROPRIEDADES LÍQUIDO CRISTALINAS VIA RADICAIS LIVRES}

\section{Por emprego de um macroagente de transferência}

A síntese de um copolímero em bloco composto de um segmento amorfo e um segmento LC a partir de um macroagente de transferência foi investigada. O poli(glicol propilênico) com terminais do tipo tiol (PPG-SH) foi usado como macroagente de transferência na polimerização de um monômero acrílico líquido cristalino contendo como mesógeno lateral o 4-(propenoilóxi-butil)oxibenzoato de 4-butilóxi-fenila ${ }^{1,30}$.
A Figura 1 demonstra a rota sintética utilizada para obtenção do copolímero em bloco poli[glicol propilênico-b-4-(propenoilóxibutil)oxibenzoato de 4-butilóxi-fenila], onde a polimerização do monômero LC foi iniciada pelo 2,2' -azo-bis-isobutironitrila (AIBN).

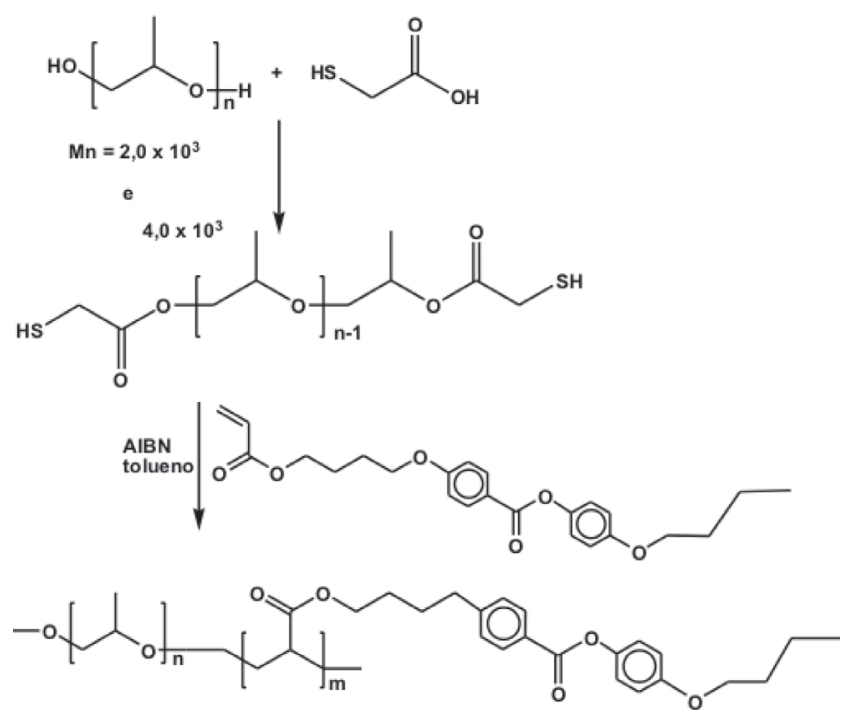

Figura 1. Rota sintética para obtenção do poli[glicol propilênico-b-4(propenoilóxi-butil)oxibenzoato de 4-butil-óxi-fenila] via radicais livres usando um macroagente de transferência com terminais do tipo tiol. Reproduzida da ref. 30, com permissão de Wiley-VCH Verlag $\mathrm{GmbH} \& \mathrm{Co} \mathrm{KG}$

Neste tipo de reação, o grupo S-H transfere o hidrogênio para uma cadeia de acrilato propagante, gerando um novo centro ativo ao longo da cadeia do poli(glicol propilênico) que, por sua vez, inicia a polimerização do monômero acrilato com conseqüente formação do copolímero em bloco.

O PPG-SH foi preparado conforme descrito na literatura ${ }^{37}$, sendo obtidos rendimentos da ordem de $80 \%$, com $\bar{M} n_{\text {rel }}$ de $1,9 \times 10^{3}$ e de $4,0 \times 10^{3}$. Na etapa da formação do copolímero foram estudadas razões molares entre o monômero acrílico e o PPG-SH de 5,3 a 53,0 , conforme a Tabela 1 . Foi observado que o copolímero onde se emprega a maior razão molar na alimentação é aquele que apresenta maior massa molar, embora o maior rendimento tenha sido para a razão molar LCP/PPG-SH de 10,1 e o copolímero que apresenta menor polidispersão é obtido quando se emprega a razão molar de 15,9 .

A Figura 2 mostra as curvas de cromatografia de exclusão por tamanho molecular (SEC) para os copolímeros formados e nenhum pico estranho ou ombros foram observados no cromatograma, indicando uma purificação eficiente dessas amostras. Este comportamento pode ser atribuído ou à extensão perfeitamente uniforme das cadeias a partir do macroagente de transferência ou é resultante de um processo de fracionamento do polímero durante a etapa de purificação.

A partir dos cromatogramas de SEC foi possível também calcular a fração de segmentos LC no copolímero, por meio de análise UV quantitativa, quando observado a $260 \mathrm{~nm}$, a $25{ }^{\circ} \mathrm{C}$ em THF, usando o homopolímero LC correspondente, para padronização do sistema cromatográfico. Observou-se que em todas as razões molares LC/PPG-SH estudadas a fração de segmentos LC é alta.

A Figura 3 mostra o espectro de ${ }^{1} \mathrm{H}-\mathrm{RMN}$ do poli[glicol propilênico-b-4-(propenoilóxi-butil)oxibenzoato de 4-butilóxifenila], produzido a partir da razão molar MLC/PPG-SH de 5,3. Esta técnica auxiliou na caracterização estrutural do copolímero a 
Tabela 1. Dados de massas molares relativas e de polidispersão obtidas por SEC para os copolímeros em bloco sintetizados via radicais livres, usando um macroagente de transferência com terminais do tipo tiol com diferentes razões molares de monômero LC/PPG-SH ${ }^{1}$

\begin{tabular}{|c|c|c|c|c|c|}
\hline Polímero & $\begin{array}{c}\text { Razão molar } \\
\text { MLC/ } \\
\text { PPG-SH }\end{array}$ & $\begin{array}{c}\text { Rendimento } \\
(\%)\end{array}$ & $\bar{M} n_{\text {rel }} \times 10^{3}$ & PDI & $\begin{array}{c}\text { Grau de } \\
\text { incorporação } \\
\text { do } \operatorname{LCM}^{\mathrm{a}}(\%)\end{array}$ \\
\hline homopolímero & - & 94,4 & 5,8 & 1,78 & 100 \\
\hline $\mathrm{a}$ & 5,3 & 90,0 & 7,1 & 1,18 & 60,2 \\
\hline $\mathrm{b}$ & 10,1 & 95,1 & 7,8 & 1,26 & 72,5 \\
\hline $\mathrm{c}$ & 15,9 & 86,7 & 12,0 & 1,02 & 81 \\
\hline $\mathrm{d}$ & 26,5 & 91,7 & 9,8 & 1,41 & 85,9 \\
\hline $\mathrm{f}$ & 53,0 & 93,6 & 16,8 & 1,56 & 98,6 \\
\hline
\end{tabular}

$\overline{\bar{M}} n_{\text {rel }}=$ massa molar numérica média relativa a padrões de poliestireno; PDI = polidispersão; solvente: tolueno; atmosfera: vácuo; temperatura: $70{ }^{\circ} \mathrm{C}$; tempo reacional : $24 \mathrm{~h} ;{ }^{\mathrm{a}} \mathrm{UV}=236 \mathrm{~nm}$.

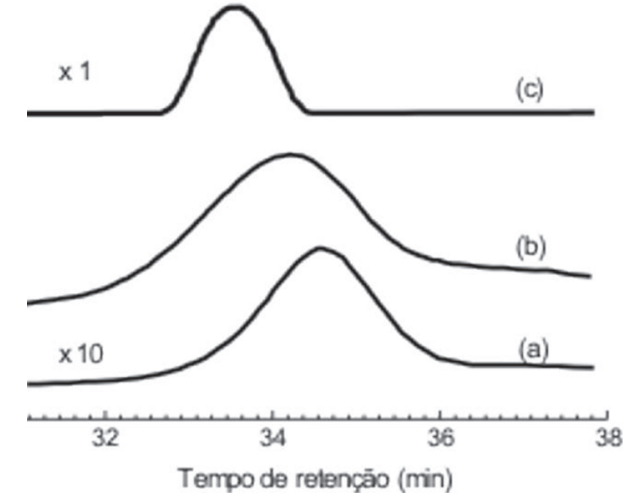

Figura 2. Curvas de SEC do poli[glicol propilênico-b-4-(propenoilóxibutil)oxibenzoato de 4-butil-óxi-fenila] obtido via radicais livres usando um macroagente de transferência com terminais do tipo tiol com diferentes razões molares MLC/PPG-SH: a) 5,3; b) 10,1; c) 15,9. Reproduzida da ref. 30, com permissão de Wiley-VCH Verlag $\mathrm{GmbH} \& \mathrm{Co} K G$

partir da identificação dos núcleos de hidrogênio presentes na porção alifática do segmento amorfo, assim como da porção aromática presente nos segmentos LC.

A partir dessa técnica foi possível também a avaliação do grau

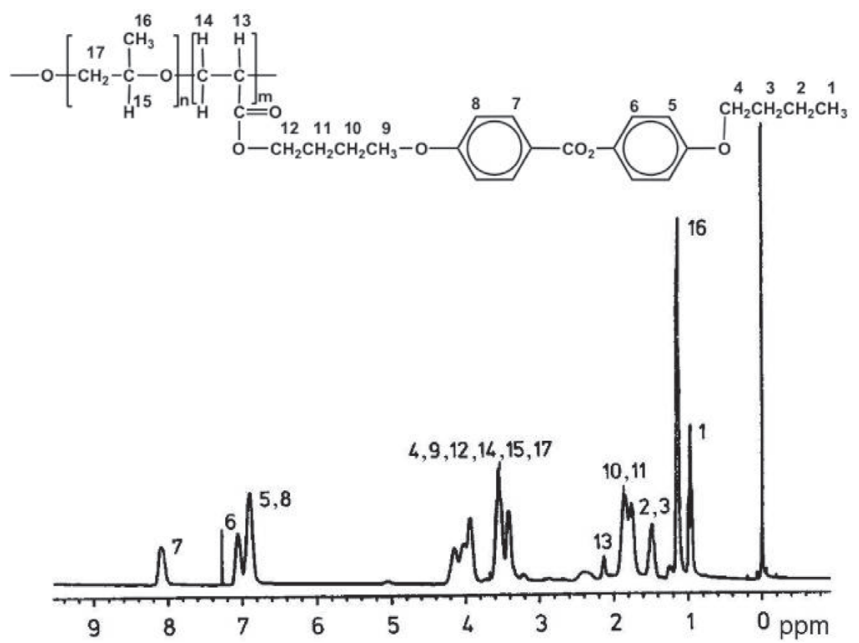

Figura 3. Espectro de ${ }^{l} H$-RMN do poli[glicol propilênico-b-4-(propenoilóxibutil)oxibenzoato de 4-butilóxi-fenila] obtido via radicais livres usando um macroagente de transferência com terminais do tipo tiol com razão molar MLC/PPG-SH de 5,3. Reproduzida da ref. 30, com permissão de Wiley-VCH Verlag $\mathrm{GmbH} \& \mathrm{Co} K G$ de incorporação de monômeros LC na cadeia por meio da relação entre áreas dos sinais característicos do segmento propilênico (especificamente, os hidrogênios ligados ao grupamento $\mathrm{C}-\mathrm{O}$, na região de 3,2 a 3,8 ppm) e do segmento LC (especificamente pelos hidrogênios aromáticos, cujos deslocamentos químicos ocorrem na faixa entre 8,0 e 8,2 ppm). Observa-se que o maior grau de incorporação é alcançado quando a razão molar de MLC/PPG-SH é de 53, sendo seguido pela razão molar de 26,5. É importante destacar que estas razões levam a valores de rendimento da ordem de $90 \%$ e massas molares também elevadas, conforme mostrado na Tabela 1 .

Os dados referentes às principais transições encontradas em curvas de DSC dos copolímeros em bloco, obtidas no segundo estágio de aquecimento, com uma velocidade de $20{ }^{\circ} \mathrm{C} / \mathrm{min}$, estão mostradas na Tabela 2, onde é possível observar que todos os copolímeros em bloco formados apresentam transições do tipo LC e que essas transições ocorrem em uma faixa de temperatura inferior à faixa de temperatura do homopolímero análogo.

Tabela 2. Comportamento térmico dos copolímeros em bloco LC estudados por $\mathrm{DSC}^{1}$

\begin{tabular}{lcc}
\hline Polímero & $\begin{array}{c}\text { Razão molar } \\
\text { MLC/PPG-SH }\end{array}$ & $\begin{array}{c}\text { Temperatura de transição } \\
\left({ }^{\circ} \mathrm{C}\right)[\Delta \mathrm{H}(\mathrm{J} / \mathrm{g})]\end{array}$ \\
\hline homopolímero & - & $\mathrm{S}_{\mathrm{A}} 113[2,7] \mathrm{N} 128[2,8] \mathrm{I}$ \\
1a & 5,3 & $\mathrm{~S}_{\mathrm{A}} 43[3,5] \mathrm{N} 75[4,4] \mathrm{I}$ \\
1b & 10,1 & $\mathrm{~S}_{\mathrm{A}} 45[2,9] \mathrm{N} 81[1,9] \mathrm{I}$ \\
1c & 15,9 & $\mathrm{~S}_{\mathrm{A}} 45[2,9] \mathrm{N} 84[2,6] \mathrm{I}$ \\
1d & 26,5 & $\mathrm{~S}_{\mathrm{A}} 45[2,0] \mathrm{N} 104[1,3] \mathrm{I}$ \\
1e & 53,0 & $\mathrm{~S}_{\mathrm{A}} 45[-] \mathrm{N} 109[-] \mathrm{I}$ \\
\hline
\end{tabular}

valores de DSC foram obtidos no segundo estágio de aquecimento; velocidade: $20{ }^{\circ} \mathrm{C} / \mathrm{min} / \mathrm{N}_{2} \mathrm{~S}_{\mathrm{A}}=$ fase esmética $\mathrm{A} ; \mathrm{N}=$ fase nemática.

Este resultado mostra-se coerente com o esperado, uma vez que o segmento glicol propilênico deve permitir maior flexibilidade da cadeia polímerica na mesofase e, com isso, as transições LC começam a acontecer em níveis energéticos mais baixos; já para a razão molar de MLC/PPG-SH de 53\%, as transições LC começam a se tornar pouco perceptíveis.

\section{Com organodissulfetos (INIFERTER)}

Os copolímeros em bloco LC foram também sintetizados pela técnica de INIFERTER, usando o dissulfeto de N,N'-tetrametiltiuram (TMTD). A Figura 4 mostra a rota empregada para obtenção do poli(estireno-b-4-bifenil 4-(4-propenoiloxi)butiloxi)benzoato de bifenila que, por sua vez, foi sintetizado pela reação de um 
macroiniciador estirênico terminado com o grupamento tiuram e um monômero metacrílico líquido cristalino, contendo como mesógeno lateral o [4-(4-propenoiloxi)butiloxi]benzoato de bifenila ${ }^{31}$.

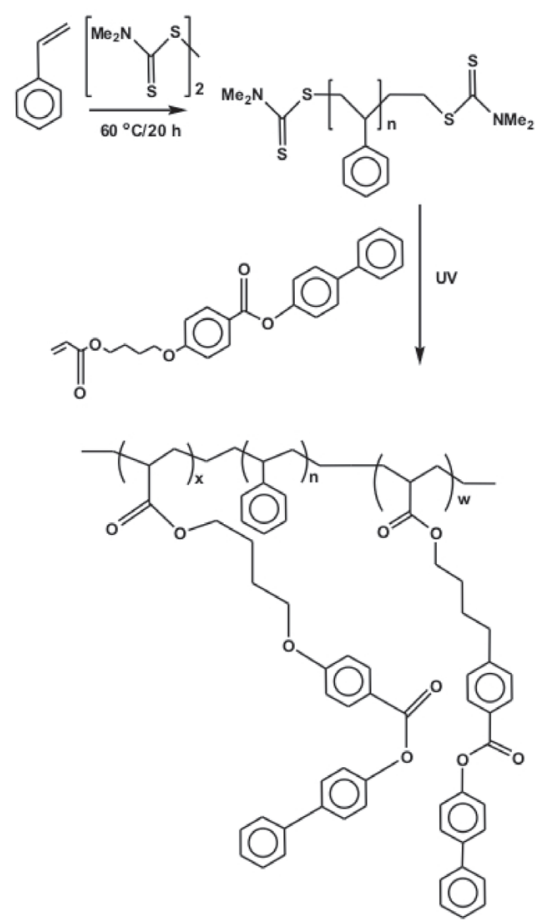

Figura 4. Rota sintética empregada para obtenção do poli\{ estireno-b-4-bifenil[4-(4-propenoiloxi)butiloxi]benzoato de bifenila\} via radicais livres usando um macroiniciador terminado por tiuram pela técnica de INIFERTER. Reproduzida da ref. 31, com permissão de John Wiley \& Sons Ltd. on behalf of the SCI

Neste tipo de polimerização, a espécie dissulfeto-orgânico apresenta um múltiplo papel. O de iniciador, o de agente de transferência e o de terminador de cadeia, sendo por isso chamada de INIFERTER. Sua química envolve a ligação carbono-enxofre (C-S) que é clivada homoliticamente, com liberação de um fragmento radical orgânico $(\mathrm{R} \bullet)$ que pode tanto iniciar uma molécula de monômero, como se adicionar a uma cadeia já em crescimento, segundo um mecanismo onde os radicais se ligam covalentemente ao terminal da cadeia, o que leva à interrupção do crescimento da mesma, de forma reversível. $\mathrm{O}$ fragmento $\mathrm{R} \cdot$ pode ser obtido a partir de energia térmica ou eletromagnética ${ }^{38}$.

A clivagem da ligação C-S nesta síntese foi feita sob radiação ultravioleta e o rendimento médio em copolímero obtido foi de cerca de $80 \%$. A análise de SEC mostrou uma diminuição no tempo de retenção do copolímero em relação ao poliestireno precursor, o que aponta para um aumento da massa molar relativa, evidenciando a extensão da cadeia polimérica pelo monômero LC, conforme observado na superposição das curvas de SEC na Figura 5. Todavia, a curva do copolímero mostra uma distribuição bimodal, apontando que as cadeias são aumentadas de maneira não uniforme pelo monômero.

A Figura 6 mostra a superposição de curvas da primeira derivada da massa contra temperatura (DTGA) de onde podem ser obtidas informações acerca da estabilidade térmica dos polímeros estudados.

Pode ser visualizado o comportamento do macroiniciador poliestireno terminado com tiuram, do homopolímero LC e do copolímero em bloco LC. Observa-se uma sutil diferença entre as temperaturas de degradação no pico, tanto para o macroiniciador

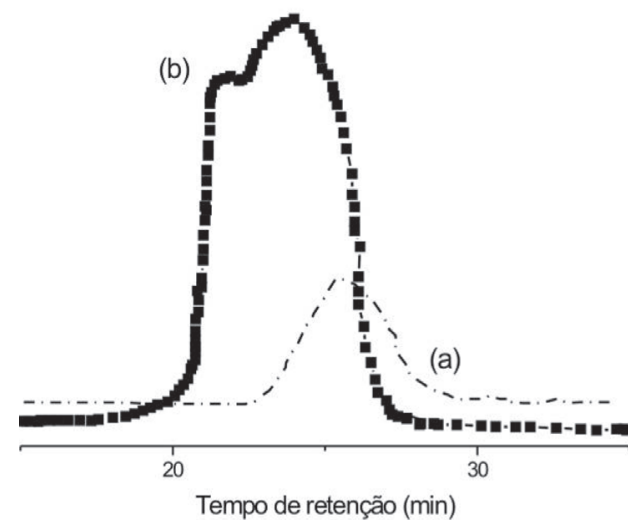

Figura 5. Superposição de curvas de SEC do macroiniciador poliestireno terminado com tiuram (a) e do copolímero em bloco LC (b) obtido via radicais livres usando um macroiniciador terminado por tiuram pela técnica de INIFERTER. Reproduzida da ref. 31, com permissão de John Wiley \& Sons Ltd, on behalf the SCI

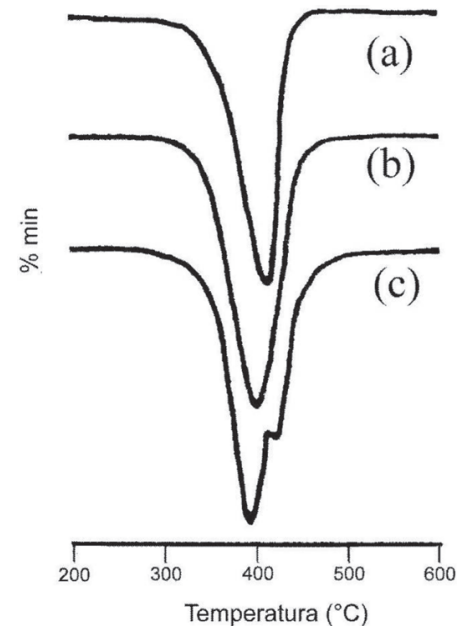

Figura 6. Superposição de curvas de primeira derivada da variação de massa contra temperatura (DTGA) do macroiniciador poliestireno terminado com tiuram (a), do homopolímero LC (b) e do copolímero em bloco LC (c) com velocidade de aquecimento de $20{ }^{\circ} \mathrm{C} / \mathrm{min}$. Reproduzida da ref. 31 , com permissão de John Wiley \& Sons Ltd, on behalf the SCI

poliestireno quanto o homopolímero LC e ambos apresentam um perfil similar entre suas curvas. No caso do copolímero em bloco, observa-se a presença de dois picos e o perfil da curva parece imitar tanto o comportamento do macroiniciador quanto do homopolímero análogo, o que pode estar relacionado à presença tanto do segmento amorfo quanto do segmento LC no material sintetizado.

A Figura 7 mostra a superposição de curvas de DSC do homopolímero LC e do copolímero em bloco contendo o segmento estirênico amorfo, tanto durante o aquecimento quanto no resfriamento.

É possível observar que a faixa de transições LC do copolímero em bloco apresenta-se numa faixa de temperatura um pouco superior à faixa de transições LC do homopolímero análogo. Observase aqui um comportamento térmico inverso ao copolímero em bloco poli[glicol-propilênico-b-4-(propenoilóxi-butil)oxibenzoato de 4-butilóxi-fenila], cuja faixa de transição LC é inferior à faixa de transição do homopolímero LC correspondente. Sendo assim, parece que o papel do bloco amorfo na mesofase pode ser modulado de acordo com a necessidade final do material. 


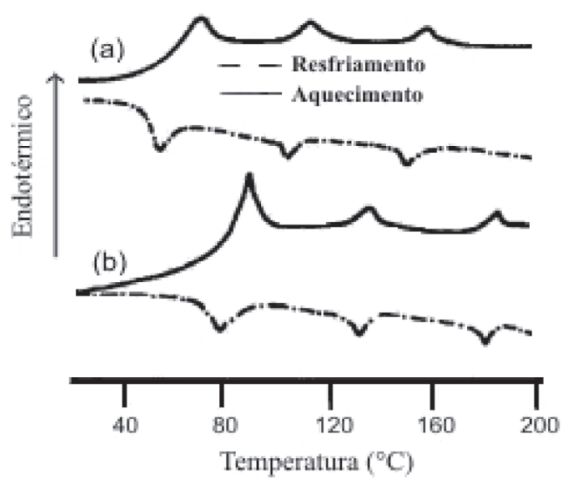

Figura 7. Curvas de DSC do homopolímero LC (a) e do copolímero em bloco $L C$ (b) com velocidade de aquecimento de $20{ }^{\circ} \mathrm{C} / \mathrm{min}$. Reproduzida da ref. 31, com permissão de John Wiley \& Sons Ltd, on behalf the SCI

Por emprego de nitróxidos estáveis do tipo $2,2,6,6$,-tetrametilpiperidino-1-oxila (TEMPO)

Os copolímeros em bloco de monômeros ordinários obtidos via radicais livres mediados por nitróxidos estáveis têm sido anunciados na literatura desde $1994^{39-50}$. A obtenção de um copolímero em bloco LC contendo um segmento amorfo e um segmento LC empregando essa técnica de polimerização foi um trabalho pioneiro do IMA. O poli\{[4-(4-propenoilóxi)butilóxi]benzoato de 4'-etilbifenila-b-estireno \} foi sintetizado a partir da formação de um macroiniciador LC obtido pela polimerização de um monômero acrílico contendo como grupamento mesogênico lateral o [4-(4propenoilóxi)butilóxi]benzoato de 4'-etil-bifenila (MILC), cuja reação foi iniciada pelo AIBN, tendo o 2,2,6,6-tetrametilpiperidino1-oxila (TEMPO ${ }^{\mathrm{MR}}$ ) como mediador da reação ${ }^{25}$.

O macroiniciador LC (MILC) produzido, quando em presença de estireno, permitiu a extensão da cadeia, formando então o copolímero em bloco com bom controle da estrutura macromolecular. A Figura 8 mostra a rota sintética empregada na preparação deste copolímero em bloco.

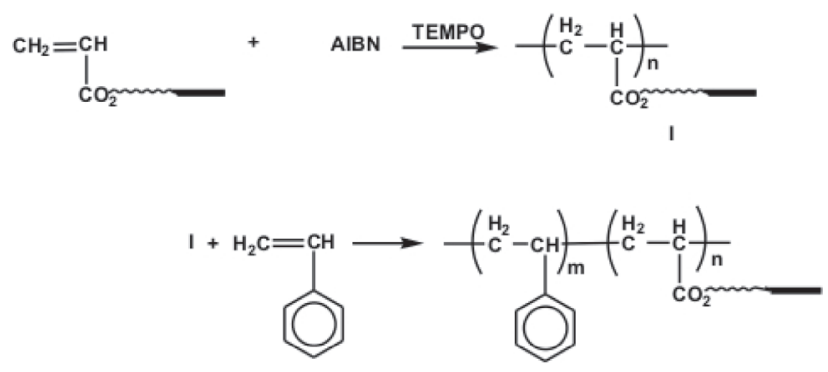

Figura 8. Rota sintética para preparação do poli[4-(4-propenoiloxi)butiloxi)benzoato de 4'-etil-bifenila-b-estireno] via radicais livres, mediada pelo TEMPO ${ }^{M R}$. Reproduzida da ref. 32, com permissão de Springer Science and Business Media

A Tabela 3 mostra as diferentes razões de iniciador (AIBN)/ terminador reversível (TEMPO ${ }^{\mathrm{MR}}$ ) empregadas na preparação do MILC. Foram observados altos valores de rendimento para todas as razões molares investigadas e uma baixa polidispersão indicando que este macroiniciador, nas condições reacionais empregadas, apresenta uma boa regularidade estrutural considerando que o mesmo foi obtido por um mecanismo via radicais livres.

A Tabela 4 mostra as características do copolímero em bloco formado a partir do emprego do macroiniciador MILC-2 sintetizado em diferentes tempos reacionais.
Tabela 3. Características do macroiniciador LC (MILC) obtido por polimerização via radicais livres mediada pelo TEMPOMR ${ }^{\mathrm{M}}$. Reproduzida da ref. 32, com permissão de Springer Science and Business Media

\begin{tabular}{|c|c|c|c|c|}
\hline $\begin{array}{l}\text { Macroiniciador } \\
\text { LC }\end{array}$ & 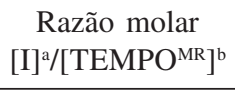 & $\begin{array}{l}\text { Rendimento } \\
(\%)\end{array}$ & $\begin{array}{l}\bar{M} n_{\text {rel }} \\
\times 10^{3}\end{array}$ & PDI \\
\hline MILC-1 & 1,2 & 78 & 6,8 & 1,49 \\
\hline MILC-2 & 1,0 & 76 & 6,9 & 1,47 \\
\hline MILC-3 & 0,83 & 76 & 6,9 & 1,47 \\
\hline
\end{tabular}

$\overline{\bar{M}} n=$ massa molar numérica média relativa a padrões de poliestireno; PDI = polidispersão, ${ }^{\mathrm{a} I}=\mathrm{AIBN}$; ${ }^{\mathrm{T}} \mathrm{TEMPO}^{\mathrm{MR}}=2,2,6,6$,-tetra-metilpiperidino-1-oxila; tempo reacional: $48 \mathrm{~h}$; solvente:1,4-dioxano, T: $135{ }^{\circ} \mathrm{C}$.

Tabela 4. Características do copolímero em bloco LC obtidos a partir da polimerização via radicais livres, iniciada pelo macroiniciador LC mediada pelo TEMPO ${ }^{\mathrm{MR}}$. Reproduzida da ref. 21, com permissão de Springer Science and Business Media

\begin{tabular}{lcccc}
\hline Polímero & $\begin{array}{c}\text { Tempo } \\
\text { reacional }(\mathrm{h})\end{array}$ & $\begin{array}{c}\text { Rendimento } \\
(\%)\end{array}$ & $\begin{array}{c}\bar{M} n_{\text {rel }} \\
\times 10^{3}\end{array}$ & PDI \\
\hline Homopolímero $^{\text {a }}$ & 24 & 81 & 23,4 & 3,77 \\
Copoli-1 $_{\text {Copoli-2 }}$ & 16 & 34 & 9,7 & 2,61 \\
Copoli-3 & 40 & 54 & 106,6 & 2,79 \\
\hline
\end{tabular}

$\overline{\bar{M}} n_{\text {rel }}=$ massa molar relativa a padrões de poliestireno; PDI = polidispersão, a reação procedida somente com AIBN e o MLC, I = MILC-1; solvente:1,4-dioxano T: $135{ }^{\circ} \mathrm{C}$.

Observou-se que os valores de rendimento aumentam à medida que se aumenta o tempo reacional, assim como os valores de massa molar relativa. Um resultado interessante e que merece maior estudo é o baixo valor de polidispersão encontrado para o tempo reacional de $64 \mathrm{~h}$. Quando se compara a técnica de radicais livres convencional com os radicais livres mediados pelo nitróxido estável observa-se um maior comprometimento no rendimento em detrimento da regularidade estrutural da cadeia.

A Figura 9 mostra a superposição dos cromatogramas de SEC do macroiniciador MILC-2 e do copolímero em bloco obtido após 16 h de reação.

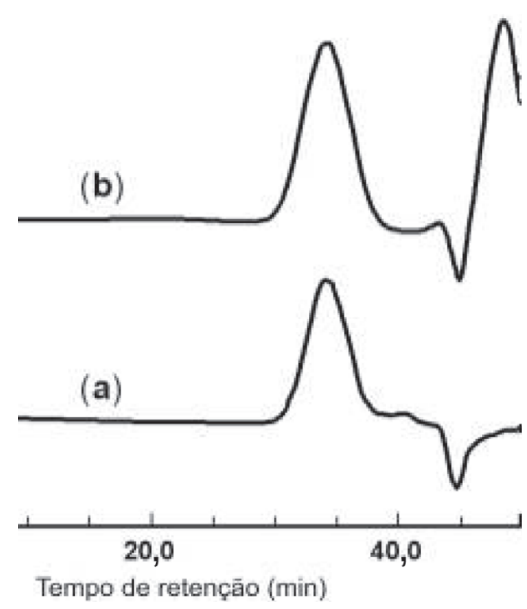

Figura 9. Superposição de cromatogramas de SEC do macroiniciador LC (MILC-2) (a) e do poli\{[4-(4-propenoilóxi)butilóxi]benzoato de 4'-etilbifenila-b-estireno\} obtido após 16 h de reação (copoli-1) (b). Reproduzida da ref. 33, com permissão de Springer Science and Business Media 
Nesta figura pode ser observada uma ligeira diminuição no tempo de retenção do copolímero em bloco quando comparados ao tempo de retenção do macroiniciador LC no mesmo sistema cromatográfico, mostrando um aumento da massa molar do copolímero. A distribuição dos tamanhos das cadeias é inferior ao obtido quando se emprega a polimerização via radical livre convencional evidenciando, assim, a potencialidade dessa técnica para obtenção de copolímeros em bloco com um melhor controle da estrutura macromolecular.

A Figura 10 mostra o comportamento térmico dos polímeros LC onde é possível observar que não há diferença significativa na faixa de transições LC entre o copolímero em bloco e do homopolímero análogo, com temperaturas de fusão cristalina em torno de $120^{\circ} \mathrm{C}$ e temperaturas de isotropização em torno de $250{ }^{\circ} \mathrm{C}$. E ainda, o elevado valor de massa molar do primeiro em relação ao último, sugere que filmes mais bem formados poderiam ser obtidos sem alteração da temperatura de formação da mesofase.

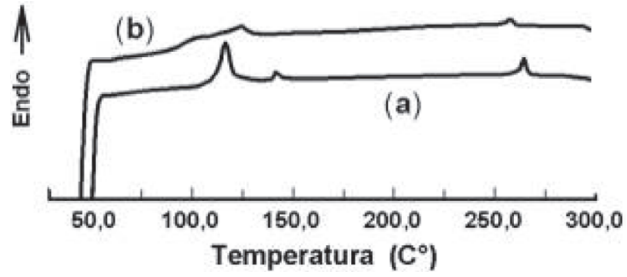

Figura 10. Curvas de DSC do macromonômero LC (MILC-2) (a) e do poli[4(4-propenoiloxi)butiloxi)benzoato de 4'-etil-bifenila-b-estireno] obtido após 16 h de reação (b). Reproduzida da ref. 32, com permissão de Springer Science and Business Media

\section{Por transferência atômica catalisada por metal de transição (ATRP)}

A potencialidade de síntese de copolímeros em bloco LC empregando o mecanismo de polimerização via radicais livres por transferência atômica catalisada por cobre foi também investigada ${ }^{33-51}$.

Essa técnica começou a ser mundialmente explorada para obtenção de copolímeros em bloco não-LC, a partir de 1995, na polimerização de monômeros estirênicos e acrílicos com bom controle estrutural. Baseia-se na capacidade de geração de uma baixa concentração de radicais livres propagantes no meio reacional devido à presença de um halogênio ou um pseudo-halogênio que atua como grupamento terminal reversível. Um complexo catalítico entre um metal de transição $\left(\mathrm{M}_{\mathrm{t}}\right)$ e um ligante $(\mathrm{L})$ é o responsável pela reversibilidade desse processo; eles formam o ciclo ativador $\left(\mathrm{M}_{\mathrm{t}}^{\mathrm{n}} / \mathrm{L}\right)$, (metal com menor número de oxidação) e desativador, $\left(\mathrm{X}-\mathrm{M}_{\mathrm{t}}^{\mathrm{n}+1} / \mathrm{L}\right)$ (onde o metal passa a apresentar maior número de oxidação $)^{51}$. $\mathrm{O}$ iniciador empregado é também de grande relevância sendo muito utilizados os do tipo haletos de alquila $^{52}$, assim como haletos de dissulfonila ${ }^{53}$.

A síntese do poli(10-\{4-[4-(2-metil-butóxi)bifenil]benzoilóxi]decila)-b-estireno), um copolímero que contém um segmento acrílico LC com fase esmética $\mathrm{C}$ quiral e um segmento amorfo, foi feita a partir da polimerização de um macromonômero acrílico líquido cristalino contendo grupamento mesogênico lateral do tipo 10-\{4-[4-(2metil-butóxi)bifenil]benzoilóxi\}decila terminado por um halogênio.

A rota sintética adotada foi a mesma utilizada na obtenção de copolímeros em bloco por polimerização via radicais livres estáveis mediada pelo TEMPO ${ }^{\mathrm{MR}}$, Figura 8. Isto é, inicialmente o macromonômero com características LC reage com um iniciador em presença de um capturador reversível de radicais livres que funciona como um mediador dos radicais que, neste caso, é o complexo catalítico (Metal/Ligante) ao invés do nitróxido estável.
O procedimento adotado para síntese do macromonômero poli(acrilato de 10-\{4-[4-(2-metil-butóxi)bifenil]benzoilóxi\}decila contendo um átomo de bromo como grupamento terminal (PMLCF$\mathrm{Br}$ ) foi efetuado usando o dicloreto de fenóxi-sulfonila (PDSC) como iniciador, e um complexo catalítico formado pelo cloreto de cobre $(\mathrm{CuBr})$ (catalisador) e a 2,2'-bipiridina (ligante) empregando condições reacionais previamente descritas na literatura ${ }^{54}$.

Os parâmetros rendimento, massa molar e distribuição de massa molar foram avaliados para dois tempos reacionais, 48 e $72 \mathrm{~h}$. A Tabela 5 mostra os resultados obtidos, onde se observa que o macroinciador LC é produzido com bom rendimento em ambos os tempos reacionais e apresenta também um bom controle da estrutura macromolecular quando esse mecanismo de polimerização é empregado.

Tabela 5. Características do macroiniciador LC (PMLCF-1-Br) obtido por polimerização via radicais livres por transferência atômica catalisada com $\mathrm{CuBr}^{36}$

\begin{tabular}{lcccc}
\hline Macroiniciador & $\begin{array}{c}\text { Tempo } \\
\text { reacional }(\mathrm{h})\end{array}$ & $\begin{array}{c}\text { Rendimento } \\
(\%)\end{array}$ & $\begin{array}{c}\bar{M} n_{\text {rel }} \\
\text { LC } 10^{3}\end{array}$ & PDI \\
\hline PMLCF-1-Br & 48 & 77 & 2,35 & 1,52 \\
PMLCF-2-Br & 72 & 75 & 2,30 & 1,50 \\
\hline
\end{tabular}

$\overline{\bar{M}} n_{\text {rel }}=$ massa molar numérica média relativa a padrões de poliestireno; PDI = polidispersão; $\mathrm{I}$ = PDSC; complexo catalítico: $\mathrm{CuBr} / 2,2$ '-bipiridina; razão molar de monômero:iniciador:catalisador:ligante de 200/12/6; solvente:1,4-dioxano; T: $130{ }^{\circ} \mathrm{C}$.

Na etapa de copolimerização, pela mistura do estireno com o macroiniciador LC (PMLCF-2-Br) com razão molar de macroiniciador/catalisador/ligante de 200/12/6, em 1,4-dioxano, observou-se a extensão da cadeia polimérica, levando à formação do poli(acrilato de 10-\{4-[4-(2-metil-butóxi)bifenil]benzoilóxi\} decila-b-estireno), que apresentou massa molar relativa de $39 \mathrm{x}$ $10^{3}$ e polidispersão de 1,9 quando o tempo reacional de $48 \mathrm{~h}$ foi empregado.

A Figura 11 mostra as curvas de cromatografia de exclusão por tamanho (SEC) para o macroinciador e o copolímero em bloco LC. Observa-se que a curva do copolímero apresenta um menor tempo de retenção em relação ao macroiniciador, também sugerindo a incorporação do estireno na cadeia.

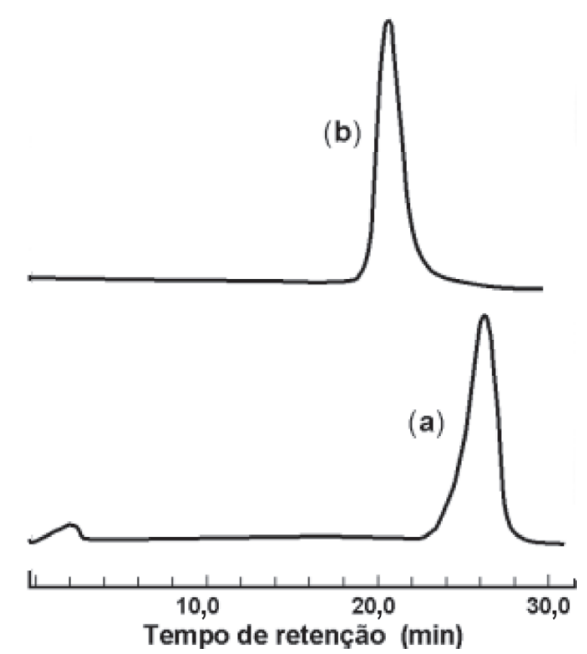

Figura 11. Superposição de cromatogramas de SEC dos polímeros LC obtidos pela técnica ATRP: a) macroiniciador PMLCF-2-Br; b) copolímero em bloco LC quiral ${ }^{36}$ 
Tabela 6. Características dos polímeros LC obtido por polimerização via radicais livres ${ }^{37}$

\begin{tabular}{lccccc}
\hline Polímero LC & Técnica & $\begin{array}{c}\text { Tempo } \\
\text { reacional }(\mathrm{h})\end{array}$ & $\begin{array}{c}\text { Rendimento } \\
(\%)\end{array}$ & $\begin{array}{c}\bar{M} n_{\text {rel }} \\
\times 10^{3}\end{array}$ \\
\hline Homopolímero $^{\mathrm{a}}$ & convencional & 48 & não informado & 27,0 & 4,40 \\
Copoli-1 $^{\text {b }}$ & ATRP & 48 & 10 & 39,0 & 1,90 \\
\hline
\end{tabular}

$\overline{\bar{M}} n_{\text {rel }}=$ massa molar numérica média relativa a padrões de poliestireno; PDI = polidispersão; ${ }^{a}$ reação procedida somente com AIBN e o MLCF;

breação procedida com razão molar de monômero:iniciador:catalisador:ligante de 200/12/6 I: MLCF-2-Br; solvente:1,4-dioxano; T: 130 C.

A massa polimérica obtida foi submetida à operação de extração empregando cicloexano como solvente e mostrou um teor de poliestireno residual de $20 \%$, juntamente com o copolímero em bloco sintetizado. A presença desse homopolímero como subproduto pode ter sido oriunda da autopolimerização do estireno na etapa de construção do segundo bloco.

Já a tentativa de copolimerização partindo-se de um macroiniciador baseado em estireno não obteve sucesso e nenhum copolímero foi alcançado $^{52}$, indicando possivelmente que neste tipo de mecanismo, macroiniciadores à base de estireno não apresentam reatividade suficiente para iniciar a polimerização de acrilatos mesogênicos.

A Tabela 6 permite comparar as características do copolímero em bloco LC obtido por um mecanismo via radicais livres controlado, por meio de transferência atômica, com aquelas de um homopolímero LC, obtido pelo sistema de polimerização via radicais livres tradicional.

Percebe-se que a formação do copolímero resulta num rendimento de apenas $10 \%$, todavia, os valores elevados de massa molar assim como sua estreita distribuição evidenciam que o mecanismo de polimerização via radicais livres por transferência atômica leva à produção de polímeros com alto grau de controle estrutural, o que, em termos de síntese de polímero LC é um grande desafio, considerando que os grupamentos funcionais que conferem a propriedade LC poderiam reagir com o grupo terminal responsável pelo crescimento da cadeia polimérica, impedindo a formação do bloco amorfo.

\section{CONCLUSÕES}

A síntese de copolímeros em bloco LC pode ser obtida por poliadição via radicais livres com controle de polidispersão, seja pelo emprego de um macroagente de transferência, por meio de nitróxidos estáveis ou pela técnica de ATRP.

A introdução de segmentos não-LC modifica o comportamento LC de copolímeros, levando à formação de mesofases em temperaturas mais baixas ou mais elevadas dependendo da flexibilidade do segmento incorporado à cadeia, o que permite a modulação da faixa de formação de mesofase no material final, de acordo com a aplicação a que se destina.

Uma comparação entre os mecanismos via radicais livres com controle de polidispersão no emprego para síntese de copolímeros em bloco mostra que o uso de um macroagente de transferência é aquele que leva a um produto final com maior rendimento (cerca de $90 \%$ ), com menor polidispersão, maior massa molar e alto grau de incorporação das unidades mesogênicas na cadeia polimérica, além de apresentar transições LC em uma faixa de temperatura inferior ao homopolímero análogo.

Quando a formação dos copolímeros em bloco se dá por emprego de nitróxidos estáveis (TEMPO) ou do tipo INIFERTER, observa-se que as reações também ocorrem com bons rendimentos e massas molares elevadas, contudo, os valores de polidispersão empregando a técnica de INIFERTER apontam para a existência de cadeias menos polidispersas que aquelas em que copolímeros em bloco são obtidos por emprego da técnica com nitróxidos estáveis.

A técnica ATRP permite a produção de polímeros com estrutura macromolecular melhor definida, além de massas molares mais altas. Contudo, quando comparada às demais, fica claro que o grande desafio recai sobre o rendimento do material, tendo em vista ser dentre todas as técnicas citadas, aquela de maior custo, tanto pela necessidade de um iniciador específico para formação do macroiniciador, quanto de reagentes com pureza elevada, seja na fase de homopolimerização ou na de copolimerização.

Em um momento onde a nanotecnologia tem sido tão buscada e os nanomateriais são alvo de pesquisas no mundo todo, conhecer o comportamento e as metodologias de síntese para produção de copolímeros em bloco com propriedades LC é estar, de fato, contribuindo para o desenvolvimento do progresso da ciência dos materiais.

\section{AGRADECIMENTOS}

À CAPES, FAPERJ, ao PADCT-FINEP, CNPq e ao CEPG/ UFRJ.

\section{REFERÊNCIAS}

1. Carvalho, K. S.; Dissertação de Mestrado, Universidade Federal do Rio de Janeiro, Brasil, 1996.

2. Walther, M.; Finkelmann, H.; Progr. Polym. Sci. 1996, 21, 951

3. Adams, J.; Gronski, W.; Macromol. Rapid Commun. 1989, 10, 553.

4. Auman, B. C.; Percec, V.; Polymer 1988, 29, 938.

5. Angeloni, A. S.; Bignozzi, M. C.; Laus, M.; Chiellini, E.; Galli, G.; Polym. Bull. 1993, 31, 387.

6. Galli, G.; Chiellini, E.; Laus, M.; Bignozzi, M. C.; Angeloni, A. S. ; Macromol. Chem. Phys. 1994, 195, 2247.

7. Serhatli, I. E.; Galli, G.; Yagci, Y.; Chiellini, E.; Polym. Bull. 1995, 34, 539.

8. Laus, M.; Bignozzi, M. C.; Fagnani, M.; Angeloni, A. S.; Galli, G.; Chiellini, E.; Francescangeli, O.; Macromolecules 1996, 29, 5111.

9. Galli, G.; Chiellini, E.; Francescangeli, O.; Ferri, D.; Wolff, D.; Springer, J.; Laus, M.; Angeloni, A. S.; Macromol. Symp. 1997, 121, 235.

10. Serhatli, E. I.; Galli, G.; Yagci, Y.; Laus, M.; Angeloni, S. A.; Chiellini, E.; Des. Monomers Polym. 1999, 2, 259.

11. Li, X. F.; Andruzzi, L.; Chiellini, E.; Galli, G.; Ober, C. K.; Hexemer, A.; Kramer, E. J.; Fischer, D. A.; Macromolecules 2002, 35, 8078.

12. Andruzzi, L.; Hexemer, A.; Li, X. F.; Ober, C. K.; Kramer, E. J.; Galli, G.; Chiellini, E.; Fischer, D. A.; Langmuir 2004, 20, 10498.

13. Pinto, M. R.; Tese de Doutorado, Universidade Federal do Rio de Janeiro, Brasil, 1996.

14. Coelho, M. R. G.; Gomes, A. S. Em Enciclopédia de guerras e revoluções do século XX; Silva, F., ed.; Campus/Elsevier: Rio de Janeiro, 2004, p. 685.

15. Fasolka, M. J.; Mayes, A. M.; Annu. Rev. Mater. Res. 2001, 31, 323.

16. Holyst, R.; Oswald, P.; Macromol. Theory Simul. 2001, 10, 1.

17. Parck, C.; Yoon, J.; Thomas, E. L.; Polymer 2003, 44, 6725.

18. Poser, S.; Fischer, H.; Arnold, M.; Prog. Polym. Sci. 1998, 23, 1337.

19. Pugh, C.; Kist, A.; Prog. Polym. Sci. 1997, 22, 601.

20. Dutta, D.; Fruitwala, H.; Kohli, A.; Weiss, R. A.; Polym. Eng. Sci. 1990, 30,1005 .

21. Barbosa, C. A.; Dissertação de Mestrado, Universidade Federal do Rio de Janeiro, Brasil, 1997.

22. Galli, G.; Chiellini, E.; Yagci, Y.; Serhatli, E. I.; Laus, M.; Bignozzi, M.C.; Angeloni, A. S.; Macromol. Rapid Commun. 1993, 14, 185.

23. Arnold, M.; Poser, S.; Fischer, R.; Frank, W.; Utschick, H.; Macromol. Rapid Commun. 1994, 15, 487. 
24. Zascheke, B.; Frank, W.; Fischer, H.; Schmutzler, K.; Arnold, M.; Polym Bull. 1991, 27, 1.

25. Kodaira, T.; Mori, K.; Macromol. Chem. 1992, 193, 1331.

26. Hefft, M.; Springer, J.; Macromol. Rapid Commun. 1990, 11, 397.

27. Percec, V.; Lee, M.; Pure Appl. Chem. 1992, A29, 723.

28. Bohnert, R.; Finkelmann, H.; Makromol. Rapid Commun. 1993, 14, 139.

29. Bohnert, R.; Finkelmann, H.; Macromol. Chem. Phys. 1994, 195, 689.

30. Gomes, A. S.; Carvalho, K. S.; Pinto, M. R.; Macromol. Rapid Commun. 1995, 16, 543 .

31. Gomes, A. S.; Barbosa, C. A.; Pinto, M. R.; Polym. Int. 1999, 48, 713

32. Barbosa, C. A.; Gomes, A. S.; Polym. Bull. 1998, 41, 15.

33. Barbosa, C. A.; Coelho, M. R. G.; Gomes, A. S.; Macromol. Symp. 2000, $168,91$.

34. Novak, K. M.; Martins, J. C. A.; Gomes, A. S.; Polym. Bull. 1996, 37, 603.

35. Novak, K. M.; Martins, J. C. A.; Gomes, A. S.; Macromol. Symp. 1997, $122,143$.

36. Barbosa, C. A.; Tese de Doutorado, Universidade Federal do Rio de Janeiro, Brasil, 2002.

37. Barbosa, R. V.; Soares, B. G.; Gomes, A. S.; J. Appl. Polym. Sci. 1993, 47, 1411.

38. Otsu, T.; Yoshida, M.; Macromol. Rapid Commun. 1982, 3, 127.

39. Georges, M. K.; Veregin, R. P. N.; Kazmaier, P. M.; Harmer, G. K.; Polym. Prepr. 1994, 35, 582.

40. Fukuda, T.; Terauchi, T.; Goto, A.; Tsujii, Y.; Miyamoto, T.; Macromolecules 1996, 29, 3050.
41. Georges, M. K.; Harmer, G.; Listigovers, K. A.; Macromolecules 1998, 31, 9087.

42. Yousi, Z.; Jian, L.; Rongchuan, Z.; Jianliang, Y.; Lizong, D.; Lansun, Z.; Macromolecules 2000, 33, 4745.

43. Yoshida, E.; Ishizone, T.; Hirao, A.; Nakahama, S.; Takata, T.; Endo, T.; Macromolecules 1994, 27, 3119.

44. Listigovers, N. A.; Georges, M. K.; Odell, P. G.; Keoshkerian, B.; Macromolecules 1996, 29, 8992.

45. Steenbock, M.; Klapper, M.; Müllen, K.; Pinhal, N., Hubrich, M.; Acta Polym. 1996, 47, 276.

46. Li, I.; Howell, B. A.; Priddy, D. B.; Smith, P. B.; Abstr. Pap. Am. Chem. Soc. 1996, S212, 612.

47. Lokaj, J.; Vlcek, P.; Kriz, K.; Macromolecules 1997, 30, 644.

48. Butz, S.; Baethge, H.; Schmidt-Naake, G.; Macromol. Rapid Commun. 1997, 18, 1049.

49. Listigovers, N. A.; Georges, M. K.; Honeyman, C. H.; Abstr. Pap. Am. Chem. Soc. 1997, S214, 410

50. Burguiere, C.; Dourges, M. -A.; Charleux, B.; Vairon, J. -P.; Macromolecules 1999, 32, 3883.

51. Matyjaszewski, K.; Macromolecules 2000, 41, 411.

52. Matyjaszewski, K.; Patten, T. E.; Xia, J.; J. Am. Chem. Soc. 1997, 119, 674.

53. Percec, V.; Kim, H.-J.; Barboiu, B.; Macromolecules 1997, 30, 6702.

54. Davis, I. K.; Matyjaszewski, K.; Macromolecules 2000, 33, 4039. 